\title{
TEMPERATURE DEPENDENCE OF THE NUCLEAR ENERGY IN RELATIVISTIC MEAN-FIELD THEORY
}

\author{
B. NERLO-POMORSKA ${ }^{1}$, K. POMORSKI ${ }^{1,2}$, J. SYKUT ${ }^{1}$ and J. BARTEL ${ }^{2}$ \\ ${ }^{1}$ Katedra Fizyki Teoretycznej, Uniwersytet Marii Curie-Sktodowskiej, Lublin, Poland \\ ${ }^{2}$ Institut de Recherches Subatomiques IN2P3-CNRS and Université Louis Pasteur, \\ Strasbourg, France
}

Received (September 25, 2004)

\begin{abstract}
Self-consistent relativistic mean-field (RMF) calculations with the NL3 parameter set were performed for 171 spherical even-even nuclei with $16 \leq A \leq 224$ at temperatures in the range $0 \leq T \leq 4 \mathrm{MeV}$. For this sample of nuclei single-particle level densities are determined by analyzing the data obtained for various temperatures. A new shell-correction method is used to evaluate shell effects at all temperatures. The single-particle level density is expressed as function of mass number $A$ and relative isospin $I$ and compared with previous estimates.
\end{abstract}

In fission dynamics and the decay of compound nuclei ${ }^{1}$ as well as in all kinds of transport theories, the proper knowledge of the nuclear single-particle level density is needed. The aim of the present paper is to determine this quantity using the RMF theory ${ }^{2}$ with the $\mathrm{NL}^{3}{ }^{3}$ set of parameters and a revised version of the Strutinsky shell correction method. ${ }^{4}$ The smooth part of nuclear energy is evaluated in our approach by an averaging in particle-number space while in the traditional Strutinsky method $^{5}$ the smoothing is performed in single-particle energy space. The advantage of the new approach consists in that the particle number is exactly conserved which was only the case on the average in the old Strutinsky method.

Mass-number and isospin dependence of the single-particle level density obtained in the RMF approach with the NL3 parameters ${ }^{3}$ were already discussed in Ref. ${ }^{6}$. Using the Strutinsky shell-correction $\operatorname{method}^{5}$ we had "removed" shell effects from the selfconsistent RMF energies in a similar way as done in Refs. ${ }^{7,8,9}$ for the Gogny hamiltonian ${ }^{10}$ and the RMF-NL3 model. ${ }^{3}$ Estimates of the macroscopic binding energies (i.e. free of shell and pairing effects) obtained in such a way at zero temperature were used as a reference to calculate the change of the total energies with temperature. To simplify the calculations, the temperature dependence of the shell-correction energy was approximated in ${ }^{6}$ by an analytical function ${ }^{11}$ instead of explicitly evaluating the Strutinsky shell correction at finite temperatures. ${ }^{12}$ 
Now, we proceed in a different and probably more consistent way, due to two changes which are, as we believe, quite essential for our study: the revised Strutinsky method, ${ }^{4}$ with a particle-number averaging instead of an energy averaging, is used to obtain the macroscopic nuclear energy. The disappearance of shell effects with increasing temperature is obtained by an energy smoothing in particles number space for each temperature separately, instead of using ${ }^{6}$ the phenomenological function of Ref. ${ }^{11}$. The comparison of the two methods and a study of the validity of the simple analytical form ${ }^{11}$ for the temperature dependence of the shell-correction energy will be given in a forthcoming publication. ${ }^{13}$

The macroscopic part of the total energy of a nucleus at temperature $T$ can be written as

$$
E_{\text {macr }}(T)=E_{S C}(T)-E_{\text {shell }}^{(n)}(T)-E_{\text {shell }}^{(p)}(T),
$$

where $E_{S C}$ is the selfconsistent energy evaluated without taking into account pairing correlations (which, anyhow, are weak or vanishing at non-zero temperatures) and $E_{\text {shell }}^{(n)}$ and $E_{\text {shell }}^{(p)}$ are the temperature dependent shell-correction energies for neutrons and protons respectively.

According to the Strutinsky prescription the shell-correction energy is given by

$$
E_{\text {shell }}=\sum_{m=1}^{\mathcal{N}} \varepsilon_{m}-\widetilde{E}(\mathcal{N}),
$$

where $\mathcal{N}$ is the number of nucleons (protons or neutrons) and $\widetilde{E}(\mathcal{N})$ is the smooth energy corresponding to a washed-out shell structure. This smooth energy can be evaluated within the traditional Strutinsky energy-averaging method ${ }^{5}$ or with the new prescription of Ref. ${ }^{4}$ in which the smoothing is performed in particle-number space $\left(\mathcal{N}\right.$-averaging). The single-particle energies $\varepsilon_{m}$ obtained within the selfconsistent RMF approach are used to evaluate the shell correction.

At higher temperatures shell effects disappear. According to Ref. ${ }^{12}$ they are negligible already at $T=2 \mathrm{MeV}$. In Ref. ${ }^{6}$ we have used the approximate $T$ dependence of the shell-correction energy given in Refs. ${ }^{14,11}$ (with $\hbar \omega=40 A^{-1 / 3} \mathrm{MeV}$ ):

$$
E_{\text {shell }}^{a p p}(T)=E_{\text {shell }}(0) \cdot \frac{\tau}{\sinh \tau}, \quad \tau=\frac{2 \pi^{2} T}{\hbar \omega} .
$$

In the present work we have replaced this phenomenological Ansatz by the shell corrections obtained by the $\mathcal{N}$-averaging method ${ }^{4}$ at different temperatures. Our prescription for the temperature dependence is then the following:

For every nucleon number $n$ in $\mathcal{N}_{\text {min }} \leq n \leq \mathcal{N}_{\text {max }}$ one evaluates the energy of the $n$-fermion system assuming a Fermi distribution for the occupation numbers of the single particle levels

$$
E(n, T)=\sum_{i=1}^{\infty} \varepsilon_{i} \nu_{i}(T)
$$


where

$$
\nu_{i}(T)=\frac{1}{1+\exp \left(\left(\varepsilon_{i}-\lambda\right) / T\right)}
$$

and where the Fermi energy $\lambda$ is found from the particle-number condition

$$
2 \sum_{i=1}^{\infty} \nu_{i}(T)=n
$$

Using the sample of $E(n, T)$ one evaluates the smooth energy $\widetilde{E}(\mathcal{N}, T)$ by performing the averaging in particle-number space (more precisely in the $n^{1 / 3}$ - space): ${ }^{4}$

$$
\widetilde{E}(\mathcal{N}, T)=\sum_{n=\mathcal{N}_{\text {min }}}^{\mathcal{N}_{\max }} \frac{2}{3 n^{2 / 3} \gamma} E(n, T) \cdot j_{6}\left(\frac{\mathcal{N}^{1 / 3}-n^{1 / 3}}{\gamma}\right)
$$

where

$$
j_{6}(u)=\frac{1}{\sqrt{\pi}} e^{-u^{2}}\left(\frac{35}{16}-\frac{35}{8} u^{2}+\frac{7}{4} u^{4}-\frac{1}{6} u^{6}\right)
$$

is the normalized sixth order Strutinsky weight function with the smearing width $\gamma=0.77$. The limits in the sum (7) are taken as $\left\{\mathcal{N}^{1 / 3} \pm 3 \gamma\right\}^{3}$. The temperature dependent shell corrections for the $\mathcal{N}$ nucleon system ${ }^{\mathrm{a}}$ is then given by the difference of (4) and (7)

$$
E_{\text {shell }}(T)=E(\mathcal{N}, T)-\widetilde{E}(\mathcal{N}, T) .
$$

These shell corrections for neutrons and protons are presented in Fig. 1 for some chains of isotopes and isotones. Subtracting them from the selfconsistent RMF-NL3 energies we obtain the macroscopic energies at various temperatures. The vanishing of the shell effects with temperature turns out to be weaker than with the previously used $^{13}$ phenomenological function.

The temperature dependence of the macroscopic part (1) of the selfconsistent energy of a nucleus at finite temperatures can be approximated by

$$
E_{\text {macr }}(T) \approx E_{\text {macr }}(0)+a T^{2},
$$

where $a$ is the so-called single-particle level-density parameter which is connected with the macroscopic nuclear entropy by the following relation

$$
S=2 a T .
$$

The level-density parameter $a$ can then be related to the macroscopic Helmholtz free energy through

$$
F_{\text {macr }}(T)=E_{\text {macr }}(T)-S T=E_{\text {macr }}(0)-a T^{2},
$$

\footnotetext{
aTo increase the accuracy of the shell-correction method the average energy dependence on the particle number given by the harmonic-oscillator sum rule ${ }^{4}$ is subtracted from (4) when evaluated (7) and then added to the average energy $\widetilde{E}(\mathcal{N}, T)$
} 

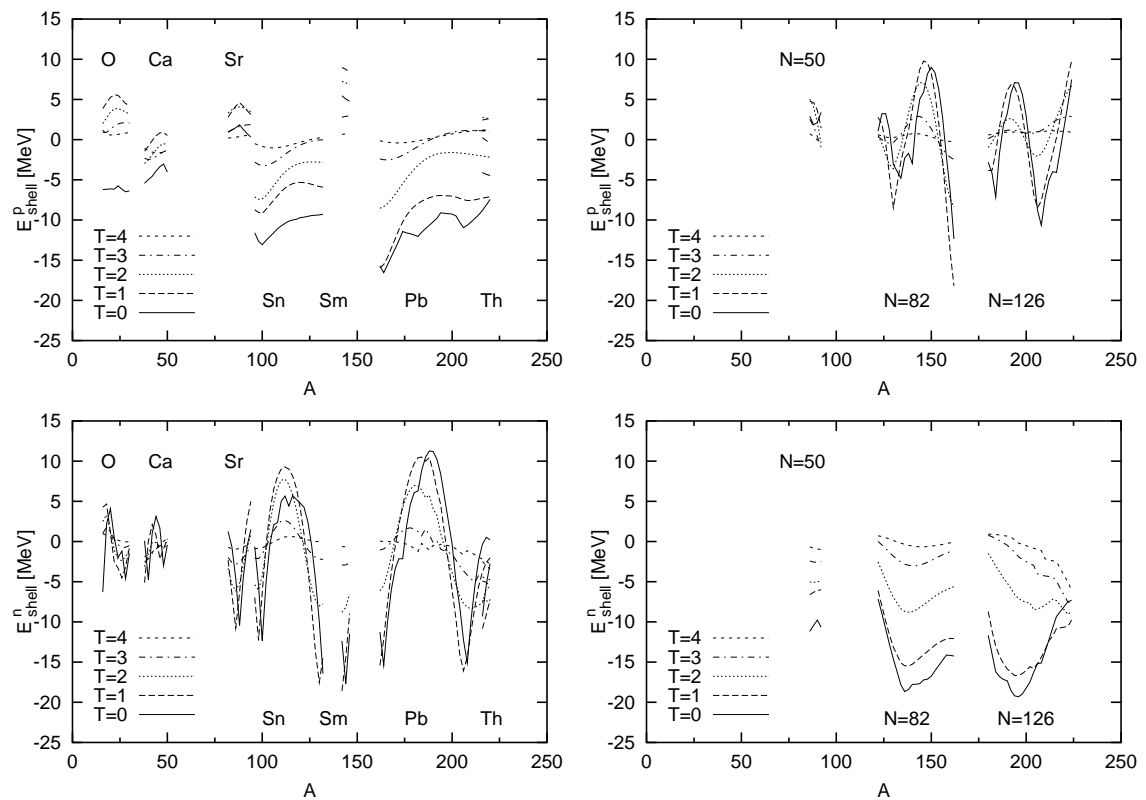

Fig. 1. The RMF-NL3N shell corrections for neutrons (top) and protons (bottom) evaluated for different temperatures as functions of mass number $A$ for isotopes (left), isotones (right).

so the knowledge of $a$ is very important in all cases where the free-energy is used, as e.g. when evaluating the adiabatic fission barriers at finite temperatures.

Usually, in a rough estimate, the level density parameter $a$ is assumed to be proportional to the mass number

$$
a=A / k \mathrm{MeV}^{-1},
$$

where $k \approx 10$. We have shown in Ref. $^{6}$ that such a simplification is too crude since the value of $a$ varies with $Z$ and $A$ as well as with the deformation of the nucleus.

One can extract the value of the level-density parameter through Eq. (10) evaluated for different nuclei at different temperatures. The $a$ values obtained at $T=1$, 2,3,4 $\mathrm{MeV}$ for the above mentioned sample of nuclei are then used to express the level-density parameter in a LDM type expansion in $A^{-1 / 3}$. We have found that the corresponding factor $k$ in (13) is not at all constant but depends on mass number $A$ and isospin parameter $I=(N-Z) / A$ of the nucleus.

The calculations were performed for 171 even-even nuclei between the drip lines which have, according to Ref. ${ }^{15}$, quadrupole moments close to zero. These are: the ${ }^{16-30} \mathrm{O},{ }^{26-30} \mathrm{Ne},{ }^{30-36} \mathrm{Si},{ }^{32-44} \mathrm{~S},{ }^{34-46} \mathrm{Ar},{ }^{38-50} \mathrm{Ca},{ }^{82-94} \mathrm{Sr},{ }^{96-132} \mathrm{Sn},{ }^{142-146} \mathrm{Sm}$, ${ }^{162-220} \mathrm{~Pb},{ }^{216-220} \mathrm{Th}$ isotopic and the $N=50$ (with $A=86-92$ ), $N=82$ (with $A=122-162$ ), and $N=126$ (with $A=180-224$ ) isotonic chains, and additional 31 spherical nuclei along the $\beta$ stability line. The neutron and proton shell corrections are shown in Fig. 1 for a few isotopic and isotonic chains. 

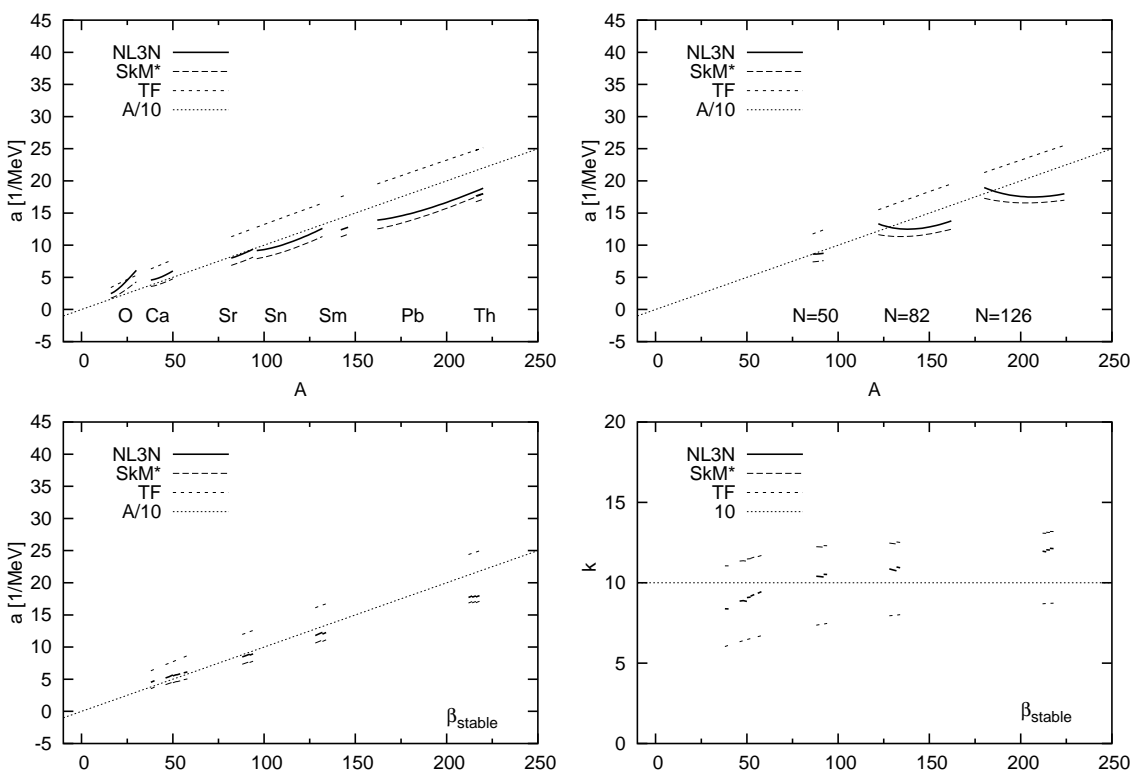

Fig. 2. Average level density parameter $a$ as function of mass number $A$ obtained in the RMFNL3N approach, as compared to the Skyrme SkM*16 and TF model ${ }^{17}$. The results are displayed for isotopes (top left), isotones (top right) and $\beta$ stable nuclei (bottom left). The $k$ values of Eq. (13) for $\beta$ stable nuclei are also shown (bottom right). The estimate of Eq. (13) is given as a dotted straight line.

Having determined the variation of $E_{\text {macr }}$ with temperature we obtain (for each nucleus separately) the level-density parameters $a(Z, A)$ through Eq. (10). This quantity is then fitted to the following mass and isospin-dependent liquid-drop type formula containing ony surface and Coulomb terms:

$$
\frac{a_{\mathrm{NL} 3 \mathrm{~N}}}{\mathrm{MeV}}=0.38 A^{2 / 3}+1.13 A^{2 / 3} I^{2}+0.0021 \frac{Z^{2}}{A^{1 / 3}} .
$$

The volume term of $a_{\mathrm{NL3N}}$ when included in such a fit turn out to be essentially zero and we have decided to omit it altogether when performing the final fit.

In Fig. 2 the values of $a$ obtained in this way for a few chains of isotopes, isotones and some $\beta$ stable nuclei are compared with the results of other models. The $a$ values obtained with the new NL3N shell-correction method ${ }^{4}$ are on the average in between the results of the TF model ${ }^{17}$ and those of the Extended Thomas-Fermi SkM* Skyrme calculations. ${ }^{16}$ The corresponding $k$ values of Eq. (13) are shown in the bottom right part of Fig. 2. For the RMF-NL3N approach one finds $7<k<12$. To guide the eye, the frequently used simple ansatz (13) is also given with $k=10$.

The following conclusions can be drawn from our analysis:

(i) The shell corrections obtained using the particle-number averaging method decrease slower with increasing temperature than those obtained with the phe- 
nomenological function (3) and survive until $T \approx 4 \mathrm{MeV}$.

(ii) The RMF-NL3N estimates of the macroscopic part of the nuclear energy change almost parabolically with temperature.

(iii) The level-density parameter $a$ deviates substantially from a simple behaviour $\left(A / 10 \mathrm{MeV}^{-1}\right)$ with a strong dependence on mass and isospin parameter which can be well approximated by a liquid-drop type expansion.

(iv) The RMF-NL3N model gives the single-particle level density closer to the TF estimates of Ref. ${ }^{17}$ than the previous RMF-NL3 approach. ${ }^{6}$

To test the quality of the single-particle level-density parameter determined here we are in the process of performing dynamical calculations (similar to those of Ref. ${ }^{1}$ ) of the decay of hot compound nuclei and confront the theoretical predictions for light-particle multiplicities and fission rates with the experimental data.

Two of us (B.N.P. and K.P.) are very grateful for the warm hospitality extended to them by the Nuclear Theory Groups of the IReS in Strasbourg. In turn J.B. greatfully acknowledges the very nice and fruitful atmosphere and good working conditions at the MCS University. This work was partially sponsored by the IN2P3Polish Laboratories Convention, Project No. 99-95.

\section{References}

1. K. Pomorski, B. Nerlo-Pomorska, A. Surowiec, M. Kowal, J.Bartel, K. Dietrich, J. Richert, C. Schmitt, B. Benoit, E. de Goes Brennand, L. Donadille and C. Badimon, Nucl. Phys. A679, 25 (2000).

2. J. Walecka, Ann. Phys. (N.Y.) 83, 491 (1974).

3. G.A. Lalazissis, J. König, and P. Ring, Phys. Rev. C55, 540 (1997).

4. K. Pomorski, Phys. Rev. C (2004) in print.

5. V.M. Strutinsky, Nucl. Phys. A95, 420 (1967).

6. B. Nerlo-Pomorska, K. Pomorski, J. Bartel and K. Dietrich, Phys. Rev. C66, 051302(R) (2002).

7. B. Nerlo-Pomorska and K. Mazurek, Phys. Rev. C66, 064305 (2002).

8. M. Kleban, B. Nerlo-Pomorska, J.F. Berger, J. Dechargé, M. Girod, and S. Hilaire, Phys. Rev. C65, 024309 (2002).

9. B. Nerlo-Pomorska, K. Mazurek, and M. Kleban, Acta Phys. Polon. B34, 1777 (2003).

10. J.F. Berger, M. Girod, and D. Gogny, Nucl. Phys. A428, 23c (1984).

11. M. Brack, R.K. Bhaduri, Semiclassical Physics, Frontiers in Physics Vol. 96 (AddisonWesley, Reading, MA, 1997).

12. M. Brack and P. Quentin, Phys. Lett. 52B, 159 (1974).

13. B. Nerlo-Pomorska, J. Sykut and J. Bartel, Proceeding of the Int. Conf. on "Atomic Nuclei at Extreme Values of Temperature, Spin and Isospin", Zakopane 2004, to be published in Acta Phys. Pol. B.

14. A. Bohr and B. Mottelson, Nuclear Structure Vol. II (Benjamin, New-York, 1975).

15. P. Möller, J.R. Nix, W.D. Myers, and W.J. Świątecki, Atom. Data and Nucl. Data Tables 59, 185 (1995).

16. C. Guet, F. Strumberger, and M. Brack, Phys. Lett. B205, 427 (1988).

17. J. Töke, and W. J. Świątecki, Nucl. Phys. A372, 141. (1981). 